\title{
Modulating the sense of agency with external cues
}

(June 2009)

Authors: James W. Moore(1), Daniel M. Wegner(2), \& Patrick Haggard(1)

(1) Institute of Cognitive Neuroscience, University College London, London, UK

(2) Department of Psychology, Harvard University, Cambridge, MA, USA

Correspondence address:

Dr. James W. Moore

Brain Mapping Unit

University of Cambridge

Department of Psychiatry

Downing Street

Cambridge CB2 3EB

Email: jm697@cam.ac.uk

Acknowledgements: JM was supported by an Experimental Psychology Society 'Study

Visit Grant' and by a UCL Bogue Research Fellowship. This work was further supported by ESRC grant RES00231571 to PH, and by NIMH Grant MH-49127 to DW.

In press, Consciousness and Cognition - With Table 1 corrected (as of Sept 2011)

Word count: 6,019 (including references, excluding figures and tables) 


\section{Abstract}

We investigate the processes underlying the feeling of control over one's actions ("sense of agency"). Sense of agency may depend on internal motoric signals, and general inferences about external events. We used priming to modulate the sense of agency for voluntary and involuntary movements, by modifying the content of conscious thought prior to moving. Trials began with the presentation of one of two supraliminal primes, which corresponded to the effect of a voluntary action participants subsequently made. The perceived interval between movement and effect was used as an implicit measure of sense of agency. Primes modulated perceived intervals for both voluntary and involuntary movements, but the modulation was greatest for involuntary movements. A second experiment showed that this modulation depended on prime-movement (temporal) contiguity. We propose that sense of agency is based on a combination of internal motoric signals and external sensory evidence about the source of actions and effects. 


\section{Introduction}

We normally have little trouble distinguishing the actions and effects that we make from those made by others. The sense we have of ourselves as individual agents capable of generating goal-directed action appears, at first sight, immediate and infallible. Two major sources of signals contributing to sense of agency have been proposed.

First, computational models of motor control suggest that the sense of agency arises principally from internal motoric signals responsible for generating the movement. In particular, an internal forward model based on efference copy may predict the sensory consequences of motor commands in advance of delayed sensory feedback (Blakemore, Wolpert and Frith, 2000; 2002). Predicted sensory information is matched against subsequent sensory information. If predicted and sensed information match, then the sensory events are self-generated, and the subject will experience a sense of agency for those events. If there is mismatch, then the sensory information describes an external event, and there is no sense of agency. This model has been used to explain the striking perceptual attenuation of self-generated stimuli (Weiskrantz, Elliot, \& Darlington, 1971; Blakemore, Wolpert, \& Frith, 2000), and pathological experiences, such as delusions of control, found in Schizophrenia. For example, Blakemore, Frith and Wolpert (2002) have suggested that the misattribution of action shown in patients experiencing delusions of control can be explained by a deficit in the internal forward model (see also Frith, 1992).

In contrast, other studies have emphasised the role of external, situational cues to agency. For example, Wegner and Wheatley (1999) induced a false sense of agency for 
movements that participants had not in fact performed. It was found that priming participants with thoughts relevant to a movement just before it was made by a confederate led participants to rate the action as self-caused. Other studies have since revealed that such consistency between prior thought and subsequent action can enhance the experience of vicarious agency for another person's actions (Wegner, Sparrow, \& Winerman, 2004), can increase agency judgments for own actions (Pronin, Wegner, McCarthy, \& Rodriguez, 2006), and even produce such effects when the prior thought is unconscious - activated through subliminal priming (Aarts, Custers, \& Wegner, 2005). These studies suggest that the sense of agency is fallible. Furthermore, the priming studies imply that the sense of agency may even occur in situations in which the participant plays no objective role in bringing about the outcome.

An account of sense of agency proposed by Wegner and Sparrow (2004) appears to accommodate these findings by suggesting that both internal and external cues contribute to the sense of agency. On this view, a processing mechanism assembles a variety of cues as to the origin of movement, from which it can then generate an account of agency. In Wegner and Sparrow's framework, these cues to agency need not be mutually exclusive. Rather, the sense of agency is based on the combination of these cues. In this way, both internal motoric signals and extrinsic information should contribute to the sense of agency. In the present study we tested this hypothesis directly using a priming paradigm to investigate how priming might alter the experience of the relation between actions and their effects. 
In each experiment we used the perceived duration of the interval between movements (key presses) and their effects (tones) as an implicit measure of sense of agency. Several studies suggest that the perceived time of self-generated actions and subsequent effects show a perceptual attraction or binding. In contrast, involuntary movements show a perceptual repulsion. This "intentional binding” effect (Haggard, Clark \& Kalogeras, 2002) is taken to be a measure of the sense of agency, because the binding between voluntary actions and effects reliably occurs in situations in which the participant is an agent (e.g. Engbert, Wohlschlaeger, \& Haggard, 2008; Tsakiris \& Haggard, 2003), relative to non-agency situations such as passive movement or the movements of other individuals. Moreover, the binding effect is modulated by the statistical relation between events (Moore \& Haggard, 2008; Moore \& Haggard, 2009) which is generally thought relevant to the perception of causation (Jenkins \& Ward, 1965)

Most studies of agency, however, have used explicit agency judgements. These involve participants introspecting upon his or her sense of agency by answering questions such as “Did you do that?” In particular, previous studies of priming (e.g. Wegner \& Wheatley, 1999; Aarts, Custers, \& Wegner, 2005) used explicit judgements to measure sense of agency. However, Synofzik, Vosgerau, and Newen (2008) recently highlighted the distinction between the feeling of agency, as captured by implicit measures, and explicit judgements of agency. Explicit judgements might be more susceptible to confounds such as prior beliefs and expectations about the task (Gawronski, LeBel, \& Peters, 2007). Moreover, Gallagher has distinguished between sense of agency and attributions of agency to individuals (Gallagher, 2007). Sense of agency refers to a first-order, prereflective experience, whilst attribution of agency refers to a second-order, reflective 
experience, that typically depends on the presence of an alternative agent. Many tasks involving explicit judgements of agency require this social attribution dimension (Wegner and Wheatley; Daprati et al., 1997). In the present experiment we therefore seek to replicate modulation of sense of agency by primes using the intentional binding measure.

Here we have used supraliminal priming to manipulate prior conscious thought about an effect. Previous research, described above, has shown that this is a valid way of modifying the content of intention prior to moving (see Wegner \& Wheatley, 1999). It is likely that such primes serve to modify the content of what Pacherie (2008) has termed 'Proximal intentions' or 'P-intentions'. P-intentions concern the goals of action (and therefore the likely consequences of movement) and are consciously accessible. Changes in the way that P-intentions are formed and sustained have been shown to influence the experience of voluntary control over an action, a key component of the sense of agency (Sebanz \& Lackner, 2007).

We have used verbal estimates of the action-effect interval as a measure of the sense of agency. The influence of consistency between prior thought and effect on binding has not previously been investigated. We predicted that primes that are congruent with a subsequent effect should enhance the sense of agency, i.e., produce lower interval estimates. Furthermore, sense of agency for voluntary movement should be relatively unaffected by primes, because the presence of intrinsic information about the movement should reduce the reliance on extrinsic cues to agency. Conversely, when the intrinsic 
motor signals are not available, as in involuntary movements, extrinsic cues should play a greater role, leading to stronger modulation of the experience of agency by effect primes.

\section{Experiment 1}

Participants made either a voluntary key press movement, or had an equivalent involuntary movement applied passively to their finger, in separate blocked conditions. All movements were followed by either a high or a low-pitched tone, at random. In addition, one of these two tones could be presented as a prime, prior to the movements. The primes aimed to induce an intention ("prior conscious thought", Wegner \& Wheatley, 1999) relating to a possible effect of the forthcoming action. The prime was either congruent or incongruent with the effect of the ensuing action. Different modes of involuntary movement induction were introduced to ensure that the experience of involuntary movement induction was not modulated by the agentic status of the inducer. For half the subjects, the involuntary passive movements were applied by the experimenter, while for the other half they were applied by a stepper motor. The presence of another agent might induce subjects to adopt an "intentional stance" (Dennett, 1987), and therefore simulate the agency of the experimenter. This in turn could lead to differences firstly in the experience of agency for involuntary movements, and secondly in the subsequent susceptibility of involuntary movements to modulation by extrinsic cues to agency. 


\section{Method}

\section{Participants}

This experiment was carried out with ethical approval, and participants gave informed consent before starting the experiment.

The original sample size was 28 , with 14 participants in each mode of movement induction condition. Three participants were excluded due to highly erratic performance on the interval estimation task (standard deviation of interval judgements above $300 \mathrm{~ms}$ across trials in one or more conditions). Note that this criterion is independent of the primary dependent variable: the mean interval estimate. Two of these excluded participants were from the motor mode of involuntary movement induction, and one from the experimenter mode of involuntary movement. The final sample therefore consisted of 25 right-handed participants (11 Females; mean age of 23 years) who took part in the experiment, which lasted 45 minutes.

\section{Procedure}

Participants gave verbal estimates of the duration of the interval between a keypress movement and a tone, in milliseconds. They were told that this interval could be between 1 and $999 \mathrm{~ms}$. In fact, only three intervals (100, 400 and $700 \mathrm{~ms})$ were presented, in a random order. There was no training beyond simple familiarisation with the apparatus. No feedback was given. 
The experimental set-up is shown in Figure 1a (for involuntary movements applied by the experimenter) and in Figure 1b (for involuntary movements applied by the motor).

Participants placed their right index finger in a Velcro strap attached to a standard keyboard. The trial structure is shown in Figure 2. First, a recorded voice told participants to "Get ready for the tone". 500ms later participants heard either a high pitched tone (frequency $1000 \mathrm{~Hz}$ ) or a low pitched tone (frequency $600 \mathrm{~Hz}$ ), played to the participant on speakers. These tones served as effect-primes. These primes could either be congruent or incongruent with the effect of actions (which were either identical high or low pitched tones).

A

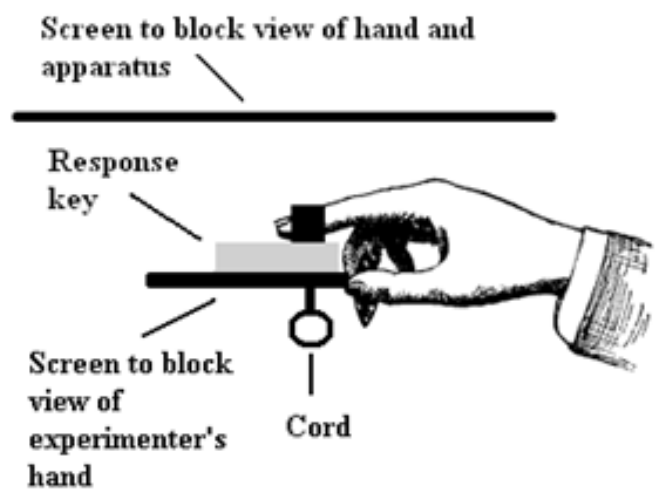

B

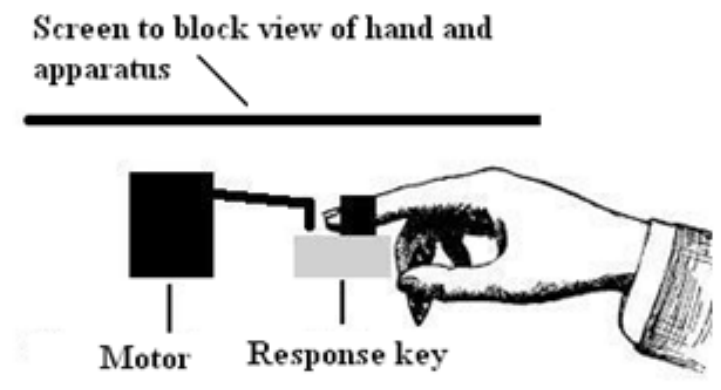

\section{Figure 1. Set up for Experiment 1. Note that involuntary movements were applied either by the experimenter (A) or a motor (B).}

Following the presentation of the first tone subjects either made a voluntary movement, or experienced an equivalent involuntary movement. Voluntary movements and involuntary movements were tested in separate counterbalanced blocks. In the voluntary movement block, subjects were instructed to press the key at a time of their choosing. 
Importantly, participants were encouraged not to press in reaction to the priming tone, but as and when they felt the urge to perform the movement. In the involuntary movement block, participants were told to relax their right index finger, and refrain from any voluntary movement. For half the participants, the experimenter then moved the participant's finger using a cord and pulley attached beneath the keyboard (see Figure 1a). The experimenter's movement caused a depression of the key to which the participant's finger was attached. This involuntary movement occurred at a time of the experimenter's choosing, which was designed to match subjects' voluntary movements. The experimenter's arm was screened from view to avoid any cues regarding onset of the involuntary movement. For the other participants, a motor above the response key was activated after a random delay following the tone. The delay was randomly drawn from a normal distribution, with a mean of $3939 \mathrm{~ms}$, and a standard deviation of $873 \mathrm{~ms}$. These parameters were obtained from participants' self-paced movements in a previous pilot version of the study. The motor briefly depressed the key before returning to its starting point; this was identical to the experimenter-induced movement condition. The motor itself was hidden from view (see Figure 1b). 


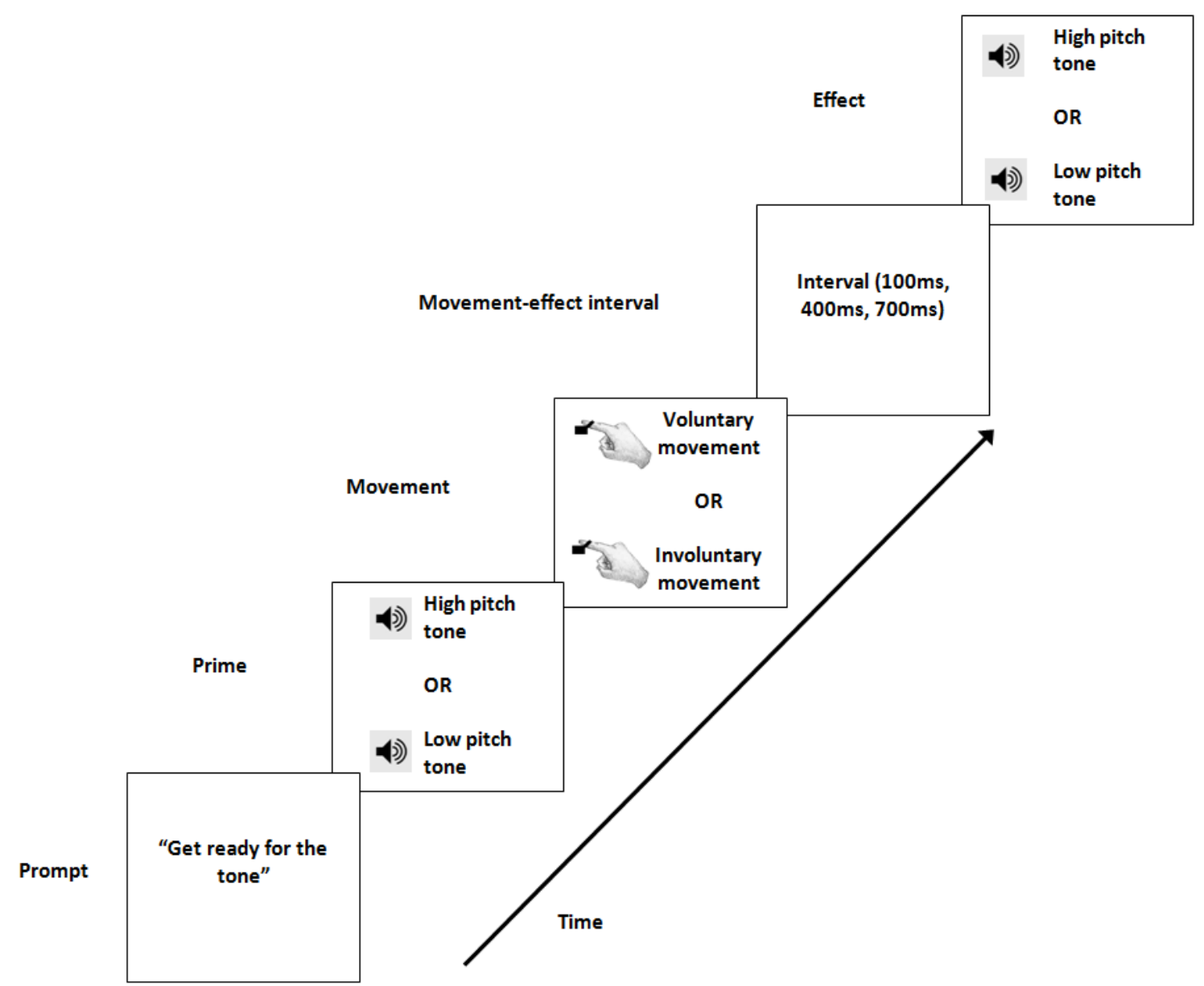

Figure 2. Experiment 1 trial structure.

A high or low tone followed each key press. The tones were identical to their prime counterparts. The interval between the movement and this second (effect) tone was either $100 \mathrm{~ms}, 400 \mathrm{~ms}$, or $700 \mathrm{~ms}$. Debriefing confirmed that none of the participants realised that only a limited range of intervals was presented.

Within each movement block, participants experienced 32 trials. Of these, 12 involved congruent primes and 12 incongruent primes. Both tone frequencies and all interval durations were equally likely, and were randomised anew for each block and subject. A further 8 trials in each block were catch trials. On these trials, subjects were asked to 
judge whether prime and effect tones were same or different, but were not asked about interval duration. They were told in advance that these catch trials would occur unpredictably. The catch trials ensured that subjects could not simply ignore primes or effects, and were designed to encourage participants to attend to the prime-effect relation.

\section{Results and Discussion}

Table 1 shows averaged interval estimates for each actual interval duration. Interval estimates are numerically lower than actual interval duration throughout. However, absolute accuracy of interval estimates varies dramatically across individuals, because people have different internal standards for time (Wearden \& Grindrod, 2003). More importantly, interval estimates systematically varied with the factors of the experimental design.

Table 1. Average interval estimates (ms) for each actual interval duration. SD across participants is shown in parentheses.

\begin{tabular}{ccccc}
\hline & & \multicolumn{3}{c}{ Actual interval length } \\
\cline { 3 - 5 } & & $\mathbf{1 0 0} \mathbf{~ m s}$ & $\mathbf{4 0 0} \mathbf{~ m s}$ & $\mathbf{7 0 0 ~} \mathbf{~ m s}$ \\
\hline $\begin{array}{c}\text { Movement } \\
\text { type }\end{array}$ & Voluntary movement & $70(35)$ & $245(116)$ & $471(176)$ \\
& Involuntary movement & $99(51)$ & $282(131)$ & $491(189)$ \\
\hline
\end{tabular}

The interval estimates increase monotonically with the actual interval, as expected. A mixed design $2 \times 2 \times 2 \times 3$ ANOVA was conducted with factors of mode of involuntary movement induction (experimenter, motor: between subjects factor), movement type (voluntary, involuntary), prime congruence (congruent, incongruent), and actual interval 
length (100ms, 400ms, 700ms). An unsurprising significant main effect of actual interval length was found, $\mathrm{F}(2,46)=139.56, \mathrm{p}<.001$. This factor did not interact with any other.

Because the actual interval length factor was not of interest, the estimates were averaged across actual intervals to provide an overall measure of binding in each condition. Figure 3 shows these averaged interval estimates plotted for the voluntary and involuntary movement conditions as a function of prime-effect relation. A mixed design $2 \times 2 \times 2$ ANOVA was conducted with factors of mode of involuntary movement induction (experimenter, motor: between subjects factor), movement type (voluntary, involuntary), prime congruence (congruent, incongruent).

There was a main effect of movement type, $\mathrm{F}(1,23),=5.07, \mathrm{p}=.034$. Intervals initiated by voluntary movements were perceived as shorter than those involving passive involuntary movements. This supports previous research showing that, self-generated actions lead to a reduction in the perceived temporal interval between movements and effects compared to non-self-generated movements (e.g. Engbert, Wohlschlaeger, \& Haggard, 2008). This is taken as a correlate of the enhanced sense of agency for selfgenerated action. 


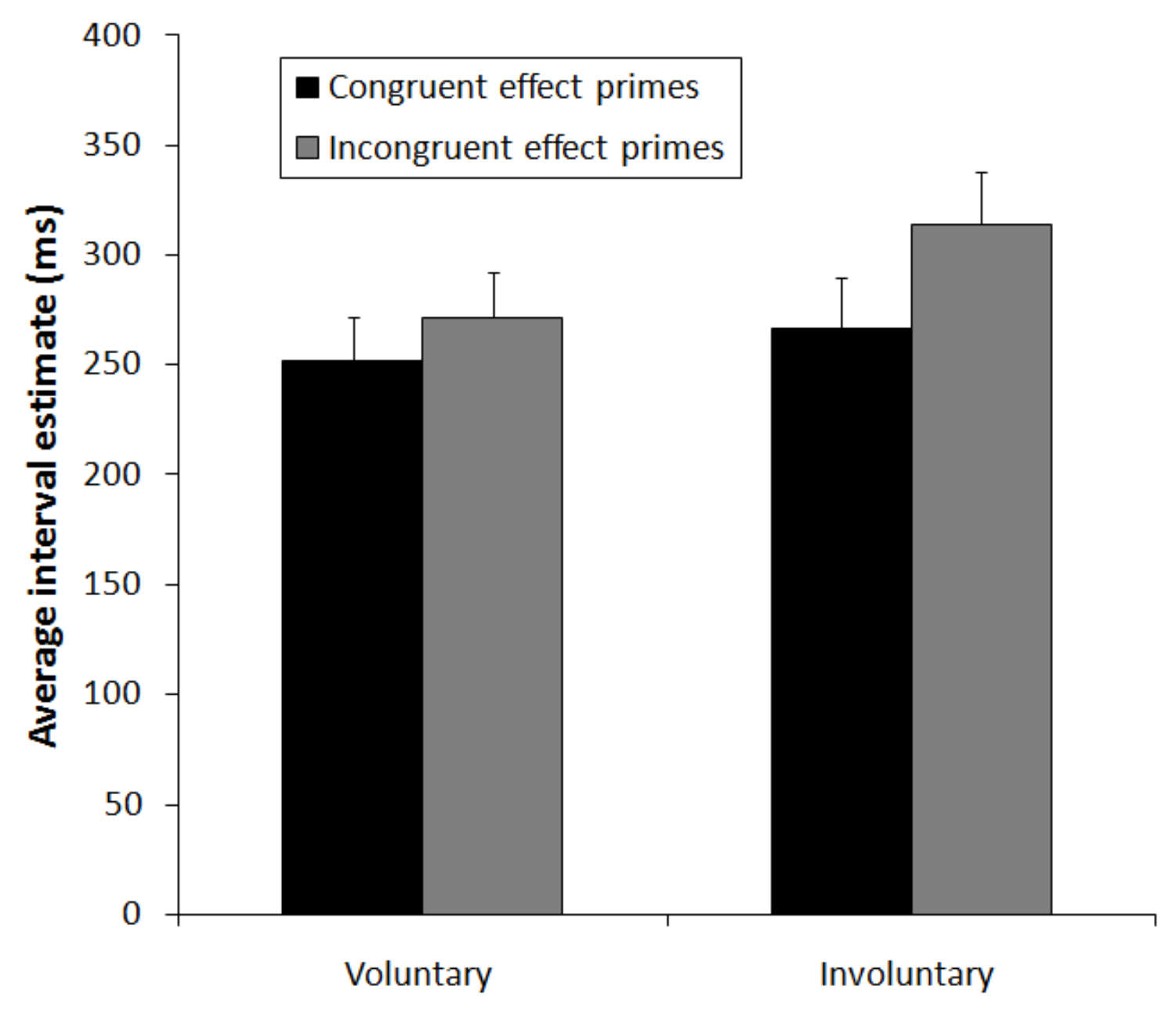

\section{Movement type}

Figure 3. Modulation of interval estimates by primes for voluntary and involuntary movements. The interval estimates are averaged across all three actual interval durations (i.e. $100 \mathrm{~ms}, 400 \mathrm{~ms}$, and $700 \mathrm{~ms}$ ). The error bars show SE across participants.

There was a significant main effect of prime congruence, $\mathrm{F}(1,23)=25.18, \mathrm{p}<.001$. This suggests that prime congruence modulated the sense of agency for both voluntary and involuntary movements, with congruent prime-effect pairing leading to an enhanced sense of agency (lower interval estimates). However, the effect of prime congruence was modulated by the type of movement, as demonstrated by the significant two-way 
interaction between prime congruence and movement type, $\mathrm{F}(1,23)=9.97, \mathrm{p}=.004$. Simple effects analysis showed that interval estimates in the involuntary movement condition were strongly modulated by the primes, $\mathrm{t}(23)=5.18, \mathrm{p}<.001$, whilst estimates in the voluntary movement condition were less strongly modulated, $\mathrm{t}(23)=2.93, \mathrm{p}=.007$ (see also Figure 3).

Finally, there was no significant main effect of mode of involuntary movement induction, $\mathrm{F}(1,23)=1.67, \mathrm{p}=.21$, and there were no significant interactions involving this factor. Therefore, inducing involuntary movements by the motor or by the experimenter's action did not affect performance. This finding rules out the possibility that any changes in the agentic experience in the involuntary movement condition were due to agency simulation.

We also calculated performance on the catch trials in this experiment. In these trials participants were asked to report whether the prime and effect tones were the same (congruent) or different (incongruent). Across all participants there were 400 catch trials in total. Overall performance on the catch trials was near perfect $(98.5 \%$ the judgements were correct), and was not systematically altered by either the mode of involuntary movement induction, or by the movement type.

Changes in the perceived interval between a movement and effect may provide implicit measures of the modulation of sense of agency. Our results suggest that conscious prior thoughts, here operationally defined by the presence of priming cues, can strengthen the experience of action-effect relations. This finding supports previous research showing 
that primes can modulate the sense of agency (e.g. Wegner \& Wheatley, 1999). However, our results also suggest that this priming effect is strongest for involuntary movements. Involuntary passive movements were more susceptible than voluntary movements to modulation by these extrinsic cues to agency.

This supports our initial prediction that the sense of agency reflects the relative strength of multiple agency cues. The sense of agency associated with voluntary movements may reflect efferent signals within the motor control system (Blakemore, Wolpert and Frith, 2000). These internal cues appear relatively resistant to modulation by effect primes. Conversely, in the absence of motor command signals, the agency processing mechanism may rely more strongly on alternative, external cues as to the source of action.

Finally, our results were similar whether the key applying passive movements to the index finger was moved by the experimenter, or by a motor under computer control. This suggests that participants' experience of their own action was not based on simulating the agency of the experimenter. Of course, this does not imply that simulation of agency plays no role in our understanding and experience of other peoples' movements.

\section{Experiment 2}

Experiment 2 investigated the importance of temporal contiguity between prime and movement in agency priming. The sense of agency may require that primes occur appropriately close in time to movement. For example, Wegner and Wheatley (1999) showed that people erroneously attributed a primed event to their own action, when it was 
in fact caused by a confederate, only if the prime occurred within a $5 \mathrm{~s}$ time window prior to movement. We investigated whether our implicit measure of agency also showed timedependent sensitivity to priming. Specifically, we predicted that perceived duration of intervals between involuntary movements and effects should be modulated more strongly by primes presented just prior to involuntary movement than by those presented longer before movement.

\section{Method}

\section{Participants}

This experiment was carried out with ethical approval, and participants gave informed consent before starting the experiment.

24 right-handed participants (9 females, 15 males; aged 16-39; mean 24 years) took part in the experiment. The procedure lasted 60 minutes.

\section{Procedure}

The procedure was based on the previous experiments. However, in Experiment 2 participants only experienced involuntary movements, and never made voluntary actions. We omitted the voluntary action condition because we found it less susceptible to priming in Experiment 1. Second, the interval between the prime and the involuntary movement was controlled to be either $1 \mathrm{~s}$ or $10 \mathrm{~s}$, at random. Involuntary movements were applied by the experimenter using a cord/ pulley (see Figure 1a). The experimenter was 
prompted to move by a visual "Go" signal at the appropriate time. This signal was not visible to the subject.

\section{Results and Discussion}

Figure 4 shows the mean interval estimates for 1s and 10s delay conditions, plotted as a function of actual interval length and prime-effect relation. A 2 x 2 ANOVA was conducted with factors of delay (1s, 10s), prime congruence (congruent, incongruent) and actual interval length. A significant main effect of prime congruence was found, $\mathrm{F}(1,23)$ $=4.74, \mathrm{p}=.04$, as in Experiment 1. This again suggests that congruent primes produce shorter interval estimates, and thus stronger binding. There was no significant main effect of the delay between prime and movement $\mathrm{F}(1,23)=.042, \mathrm{p}=.84$. 


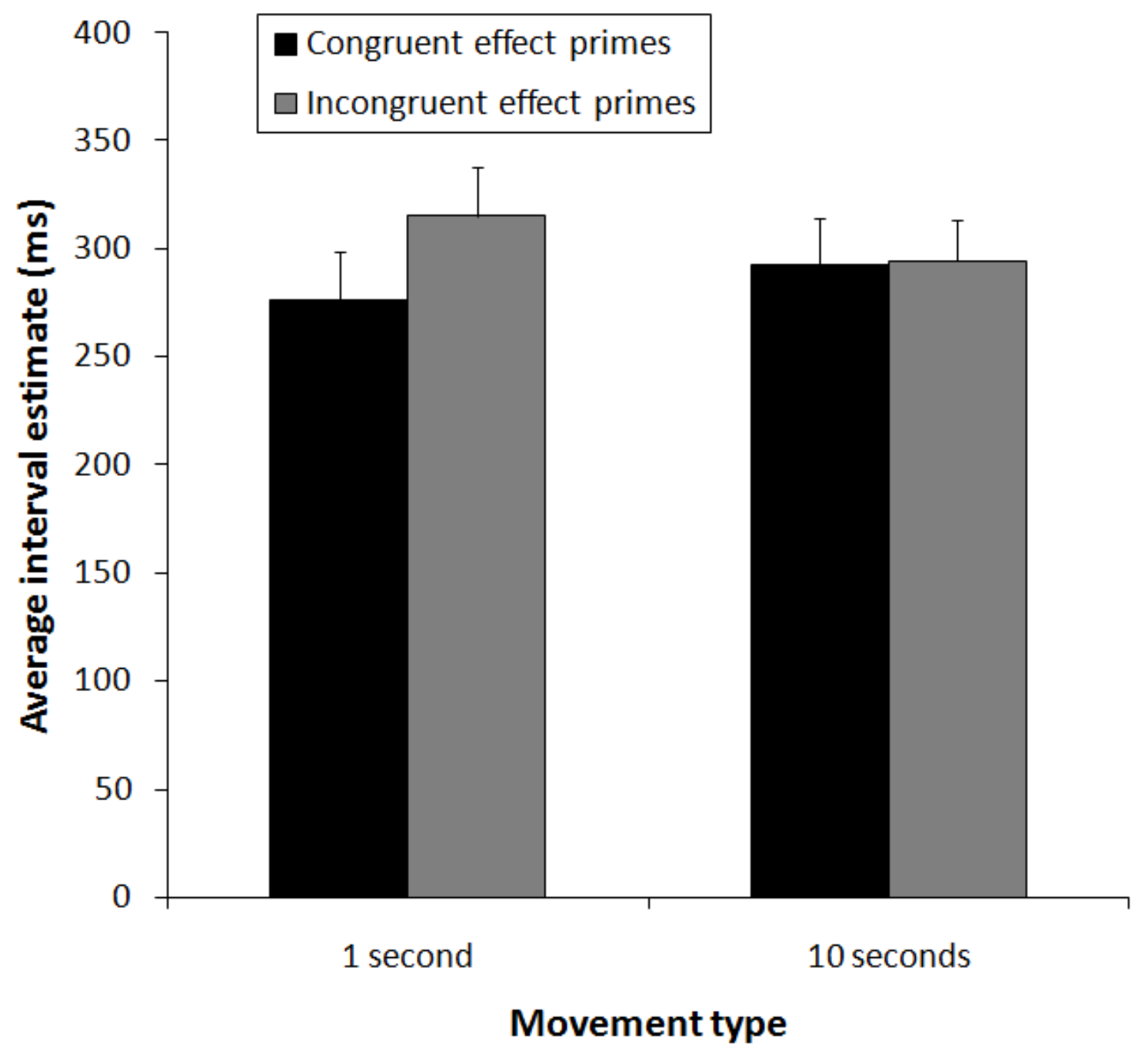

Figure 4. Modulation of the sense of agency by effect primes at 1s prior to involuntary movement onset and 10s prior to involuntary movement onset. The interval estimates are averaged across all three actual interval durations (i.e. 100ms, 400ms, and 700ms). The error bars show SE across participants.

Crucially, there was also a significant delay $\mathrm{x}$ prime congruence interaction, $\mathrm{F}(1,23)=$ $5.10, \mathrm{p}=.034$. Simple effects analysis showed that interval estimates in the 10 s delay condition were scarcely affected by prime congruence, $\mathrm{t}(23)=.128, \mathrm{p}=.90$, while 
estimates in the $1 \mathrm{~s}$ delay condition were strongly modulated, $\mathrm{t}(23)=4.75, \mathrm{p}<.001$ (see also Figure 4).

Although participants in Experiment 2 never made active movements, we replicated the finding of Experiment 1 that priming action effects can influence the experience of the action-effect relation in a way that reproduces the action-effect binding component of the sense of agency. This suggests that priming might induce some aspects of the sense of agency when agency is in fact entirely absent. Previous studies focussed on ambiguous situations where agency was possible, but found priming effects on trials where agency did not in fact occur (Wegner \& Wheatley, 1999; Aarts, Custers, \& Wegner, 2005). More importantly, we showed that the priming effect depends on the temporal relation between prime and involuntary movements. Priming the effect of an action only imparted the binding component of the sense of agency to involuntary movements when prime and involuntary movement are sufficiently close (1s), and not at longer delays (10s). This finding replicates Wegner and Wheatley's result showing the importance of temporal contiguity between prime and movement in the modulation of agency by those primes (Wegner \& Wheatley, 1999). It suggests that the agency processing mechanism does indeed infer agency from external cues, but does so only within a relatively circumscribed time window.

\section{General Discussion}

We used the perceived duration of intervals between actions and effects as an indirect measure of the sense of agency. We replicated previous results showing that intervals 
initiated by self-generated actions are perceived as shorter than intervals initiated by other events, in this case involuntary movements (e.g. Engbert, Wohlschlaeger, \& Haggard, 2008). Effect primes significantly modulated the experience of agency, and did so significantly more for involuntary than for voluntary movements.

\section{Alternative interpretations and issues}

In these experiments we compared modulation of intentional binding by primes for voluntary and involuntary movements. We cannot rule out the possibility that participants were occasionally, or very slightly active in the involuntary condition, despite our instructions to remain passive. In that case, the interaction of Experiment 1 would relate to level of motor or proprioceptive activity rather than its presence/absence. However, this possibility is not a fundamental challenge to our results. Firstly, the instruction not to move is not uncommon or particularly problematic, either in the experimental laboratory, or in everyday life. We had no reason to suspect that participants were not following this instruction. Furthermore, even if there were occasional micro-movements, the level of motor activity would still be very different in the two conditions, because it was sufficient to cause a keypress in the active condition, but not in the passive condition.

Finally, there is an inherent limitation in the method used to determine action-effect binding in this study. Interval estimates cannot tell us whether the differences in binding between active and passive movements reflect differences in perception of both actions and tones, or just one of these. We chose this method for two reasons. Firstly, it overcomes some methodological concerns with temporal perception of single events. 
Secondly, it necessarily requires participants to represent the action-effect relation that lies at the heart of the sense of agency (Engbert, Wohlschlaeger, \& Haggard, 2008).

\section{Sense of agency and agency cues}

Our experiments tested Wegner and Sparrow's (2004) suggestion that the sense of agency depends on a mechanism that uses multiple cues as to the origin of actions and their effects. These experiments showed that, in general, effect primes can modulate the sense of agency. However, a novel finding of our study is that extrinsic cues are most effective for involuntary movements. Where an internal agency cue exists within the motor system (as in voluntary movements) alternative external cues to agency have a reduced impact on action experience. However, where no such internal cue exists (as in the case of involuntary movement) conscious intentions evoked by primes assume a much greater role in the generation of the sense of agency.

Our results suggest that some aspects of the sense of agency (in our study, the actioneffect binding component of sense of agency) can be induced in passive movements where actual voluntary motor commands are absent. At first sight this may seem contradictory. However, our results replicate and extend previous data showing that participants may experience agency for actions and effects they do not control (Aarts, Custers, \& Wegner, 2005; Wegner \& Wheatley, 1999) and even for the movements of others (Wegner, Sparrow, \& Winerman, 2004). Furthermore, our results demonstrate this fallibility of sense of agency using an implicit and indirect measure as opposed to an explicit judgement of agency (for a discussion of implicit and explicit aspects of agentic experience see Synofzik, Vosgerau, \& Newen, 2008). Critically, the results also show 
that this fallibility is reduced when the movement is actually under voluntary control. External cues to agency play a reduced role when intrinsic motor commands are present. This suggests that the sense of agency is generated by a combination of both intrinsic and extrinsic cues to agency.

However, the present study suggests that intentional binding (and by extension the sense of agency in general) is heavily constrained by intrinsic motor signals: primes had less effect on the perception of keypress-tone intervals for active motor keypresses than for passive movements. This resulted in the observed interaction between voluntary and involuntary movement. It is also consistent with previous research shows the special contribution of intrinsic motor information to intentional binding (e.g. Haggard, Clark \& Kalogeras, 2002; Engbert, Wohlschlaeger, \& Haggard, 2008).

It should be noted that this pattern of results might not be due solely to differences in the contribution of intrinsic motor signals. For example, changes in proprioception generated by the different types of movement may also contribute to the observed interaction between voluntary and involuntary movement in Experiment 1. In this way, proprioception is likely to be another cue that carries information as to the agentic source of movements. Future research should try and quantify the relative contributions of such cues to sense of agency.

\section{Cue integration and sense of agency}

How might intrinsic and extrinsic cues to agency integrated? Some form of Bayesian cue integration (e.g. Hendricks, Wiggers, Jonker, Haselager, 2007; Lau, Rogers, \& 
Passingham, 2007) seems likely. On this view the sense of agency would be determined by an optimal combination of different cues. Our study focussed on intrinsic cues to agency in the form of efference copy of motor commands and extrinsic cues to agency in the form of primes. In voluntary action, intrinsic information would receive higher weighting, presumably because efference copy provides highly reliable temporal information (Tsakiris, Haggard, Franck, Mainy, \& Sirigu, 2005), and primes would receive lower weighting. This would explain the reduction in modulation by primes for voluntary movements. Conversely, in involuntary, passive movement, there is no intrinsic cue to agency, and extrinsic cues are strongly weighted. This would explain the enhanced modulation of sense of agency by primes for involuntary movements.

An interesting corollary of this Bayesian framework is that sufficient weighting of extrinsic information should, in principle, be able to overwrite the intrinsically generated sense of agency. A motor command to perform action A might be over-ridden by extrinsic information that one had performed action B. External evidence might persuade people that they had performed voluntary actions when they in fact did not. This prediction could have interesting legal implications, given the link between voluntary control and responsibility (Haggard, 2008).

This overwriting of intrinsically generated information has previously been demonstrated for agency processing (e.g. Wegner \& Wheatley, 1999; Wegner, Sparrow, \& Winerman, 2004). It has also been strikingly demonstrated in the context of decision making with the ‘choice blindness' effect (e.g. Johansson, Hall, Sikström, \& Olsson, 2005). Participants viewed photographs of two separate faces, and pointed to the one they found most 
attractive. The 'chosen' photograph was then given to the participant, who then had to justify their choice. Surreptitiously, the experimenter manipulated the relationship between the chosen photograph (the one pointed to) and the one presented, such that there were mismatches. Interestingly, they found that participants would accept, and even justify, a choice which they had not in fact made. This intrinsically generated choice may have been sufficiently weak in relation to the extrinsic cue (in the form of the presented photograph), resulting in a reversal of participants' perception of what they had actually chosen.

In general terms, our proposal suggests that the sense of agency cannot be fully captured by properties of the motor control system, as proposed by the traditional 'comparator' model (e.g. Frith, 1992; Blakemore, Wolpert \& Frith, 2002). The limitations of the comparator models have been articulated elsewhere (e.g. Synofzik, Vosgerau \& Newen, 2008). Furthermore, the framework we propose offers testable predictions. For example, if the experience of our actions depends on a process of cue integration, then neuropsychological principles predict that some patients should have abnormal and inappropriately integrated experiences of action, due to lesions that compromise the integration process. Indeed, recent studies of anosognosia for hemiplegia (Berti et al., 2005; Fotopoulou et al., 2008) confirm these predictions. These patients experience making actions with their affected limb, despite being completely unable to move it due to plegia. Their experience of action appears to be based solely on their intention to move the affected limb, and does not integrate the sensory, visual and external evidence that the limb has not moved. We would predict that such patients would show no priming effect at all in our paradigm. This prediction could be tested in future studies. 
Finally, Experiment 2 confirmed that temporal contiguity between prime and action plays a key role in agency (see Wegner \&Wheatley, 1999). Temporal contiguity between action and effect plays an important role in agency processing (Haggard, Clark, \& Kalogeras, 2002). More generally, contiguity between causes and effects is a powerful cue to causality (Michotte, 1963). The time window over which effect priming works may reflect the maximum thought-action delay that could still support an inference of agency, and may also correspond to the typical interval of preparation for voluntary actions. In the present study, we have studied only a time-point close to movement at which priming is effective, and an earlier one at which it is not. A clearer picture of the time course of this effect would need more complete sampling of prime-action intervals.. Moreover, the sense of agency does not require that prior intentional states are explicitly represented in consciousness. There are frequent instances in which we have a sense of agency for relatively automatic movements that seem to occur in the absence of prior conscious thought. Indeed, sense of agency can occur for primes that are not consciously detected (Aarts, Custers, \& Wegner, 2005).

In summary, our study suggests that sense of agency, at least for extracorporeal nonbodily effects of action, is not simply a direct corollary of the voluntary motor commands. Instead, it is based on a mechanism that combines a both internal motoric cues and external sensory cues about the relation between actions and their effects. The sense of agency can be modulated by extrinsic cues, but this effect is much stronger when no genuine motor signal is available. When a person does in fact control an action, the same level of external evidence is less effective in modulating the sense of agency, and 
perceptual experience depends largely on the voluntary motor command itself. A Bayesian cue integration process may weight the contribution to the sense of agency from voluntary motor commands and from external situational evidence in any particular context. 


\section{References}

Aarts, H., Custers, R., \& Wegner, D. M. (2005). On the inference of personal authorship: Enhancing experienced agency by priming effect information. Consciousness and Cognition, 14, 439-458.

Berti, A., et al. (2005). Shared cortical anatomy for motor awareness and motor control. Science, 309, 488-491.

Blakemore, S-J, Wolpert, D. M., \& Frith, C. D. (2000). Why can't you tickle yourself? Neuroreport, 11, 11-16.

Blakemore, S-J, Wolpert, D. M., \& Frith, C. D. (2002). Abnormalities in the awareness of action. Trends in Cognitive Sciences, 6(6), 237-242.

Daprati, E., Frank, N., Georgieff, N., Proust, J., Pacherie, E., Dalery, J., and Jeannerod, M. (1997). Looking for the agent: an investigation into consciousness of action and self-consciousness in schizophrenic patients. Cognition, 65, 71-86.

Dennett, D. (1987). The Intentional Stance. Cambridge, Mass.: MIT Press

Engbert, K. Wohlschlaeger \& Haggard, P. (2008). Who is causing what? The sense of agency is relational and efferent-triggered. Cognition, 107(2), 693-704.

Fotopoulou, A., Tsakiris, M., Haggard, P., Vagopoulou, A., Rudd, A., \& Kopelman, M. (2008). The role of motor intention in motor awareness: an experimental study on anosognosia for hemiplegia. Brain, 131(12), 3432-3442.

Frith, C. D. (1992). The Cognitive Neuropsychology of Schizophrenia. Hove: Lawrence Erlbaum Associates.

Frith, C. D. (2005). The self in action: Lessons from delusions of control. Consciousness and Cognition, 14, 752-770. 
Gallagher, S. (2007). Sense of agency and higher-order cognition: Levels of explanation for schizophrenia. Cognitive Semiotics, 0, 32-48.

Gawronski, B., LeBel, E. P., \& Peters, K. R. (2007). What do implicit measures tell us? Scrutinizing the validity of three common assumptions. Perspectives on Psychological Science, 2, 181-193.

Haggard, P. (2005). Conscious intention and motor cognition. Trends in Cognitive Sciences, 9, 290-295.

Haggard, P. (2008). Human volition: towards a neuroscience of will. Nature Reviews Neuroscience, 9, 934-946.

Haggard, P., Clark, S., \& Kalogeras, J. (2002). Voluntary action and conscious awareness. Nature Neuroscience, 5(4), 382-385.

Hendricks, K. V., Wiggers, P., Jonker, C. M., \& Haselager, W. F. (2007). Towards a computation model of the self-attribution of agency. In P. Oliver and C. Kray (Eds.), Proceedings of the Artificial Intelligence and Simulation of Behaviour Annual Convention (pp. 350-356).

Hume, D. (1888). A treatise of human nature. In L. A. Selby-Bigge (Ed.), Hume's Treatise of human nature. Oxford: Oxford University Press, Clarendon Press. (Original work published 1739).

James, W. (1890). The Principles of Psychology, London; MacMillan

Jenkins, H. M., \& Ward, W. C. (1965). Judgment of contingency between responses and outcomes.Psychological Monographs, 79 (whole volume X).

Johansson, P., Hall, L., Sikstrom, S., \& Olsson, A. (2005). Failure to detect mismatches between intention and outcome in a simple decision task. Science, 310, 116-119. 
Lau, H. C., Rogers, R. D., and Passingham, R. E. (2007). Manipulating the experienced onset of intention after action execution. Journal of Cognitive Neuroscience, 19, $1-10$.

Michotte, A. (1963). The perception of causality (trans. T. R. Miles \& E. Miles). New York: Basic Books.

Moore, J. W., \& Haggard, P. (2008). Awareness of action: Inference and prediction. Consciousness and Cognition, 17(1), 136-144.

Moore, J., Deal, D. C., Haggard, P., \& Lagnado, D. (2009). Feelings of control: Contingency determines the human experience of action. Cognition, 110(2), 279283.

Pacherie, E. (2008). The phenomenology of action: A conceptual framework. Cognition, 107, 179-217.

Pronin, E., Wegner, D. M., McCarthy, K., \& Rodriguez, S. (2006). Everyday magical powers: The role of apparent mental causation in the overestimation of personal influence. Journal of Personality and Social Psychology, 91, 218-231.

Sebanz, N. \& Lackner, U. (2007). Who's calling the shots? Intentional content and feelings of control. Consciousness and Cognition, 16(4), 859-876

Synofzik, M., Vosgerau, G., \& Newen, A. (2008). Beyond the comparator model: a multifactorial two-step account of agency. Consciousness and Cognition, 17(1), 219-239.

Tsakiris M, Haggard P, Franck N, Mainy N \& Sirigu A (2005). A specific role for efferent information in self-recognition. Cognition, 96(3), 215-231.

Wearden, J. H., \& Grindrod, R. (2003). Manipulating decision processes in the human scalar system. Behavioural Processes, 61, 47-56. 
Weiskrantz, L,, Elliott, J., \& Darlington, C. (1971). Preliminary observations of tickling oneself. Nature, 23, 598-599.

Wegner, D. M. (2002). The Illusion of Conscious Will. Cambridge, MA: MIT Press.

Wegner, D. M. \& Sparrow, B. (2004). Authorship processing. In M. Gazzaniga (Ed.), The Cognitive Neurosciences III (pp. 1201-1209). Cambridge, MA: MIT Press.

Wegner, D. M., Sparrow, B., \& Winerman, L. (2004). Vicarious agency: Experiencing control over the movements of others. Journal of Personality and Social Psychology, 86, 838-848.

Wegner, D. M. \& Wheatley, T. (1999). Apparent mental causation: sources of the experience of will. American Psychologist, 54(7), 480-492. 